\title{
A Preliminary Study on Piezo-aeroelastic Energy Harvesting Using a Nonlinear Trailing-Edge Flap
}

\author{
Jae-Sung Bae* \\ School of Aerospace and Mechanical Engineering, Korea Aerospace University, Goyang-si 10540, Gyeonggi-do, Korea
}

Daniel J. Inman**

Department of Aerospace Engineering, University of Michigan at Ann Arbor, Ann Arbor MI 48109-2140, USA

\begin{abstract}
Recently, piezo-aeroelastic energy harvesting has received greater attention. In the present study, a piezo-aeroelastic energy harvester using a nonlinear trailing-edge flap is proposed, and its nonlinear aeroelastic behaviors are investigated. The energy harvester is modeled using a piezo-aeroelastic model of a two-dimensional typical section airfoil with a trailing-edge flap (TEF). A piezo-aeroelastic analysis is carried out using RL and time-integration methods, and the results are verified with the experimental data. The linearizing method using a describing function is used for the frequency domain analysis of the nonlinear piezo-aeroelastic system. From the linear and nonlinear piezo-aeroelastic analysis, the limit cycle oscillation (LCO) characteristics of the proposed energy harvester with the nonlinear TEF are investigated in both the frequency and time domains. Finally, the authors discuss the air speed range for effective piezo-aeroelastic energy harvesting.
\end{abstract}

Key words: Piezoaeroelasticity, nonlinear aeroelasticity, energy harvester, piezoaeroealstic energy harvesting, control surface

\section{Introduction}

Over the past two decades, engineering researchers have increasingly become interested in energy harvesting. The goal of energy harvesting is to provide electrical power for low-powered wireless applications and/or to recharge a storage device such as a battery. For various energy sources, the conversion of mechanical energy like vibrations into electricity has been one of the most popular topics among those studying energy harvesting $[1,2]$. Many researchers have focused on transforming mechanical vibrations into electricity using piezoelectric, electromagnetic, and electrostatic transduction mechanisms.

One energy harvesting source available in the vicinity of remotely operated systems is the aeroelastic vibrations that result from interaction with airflow. An energy harvester would enable a remotely operated system to function anywhere that airflow is available. Over the past decade, many researchers have focused on exploiting piezoaeroelastic energy harvesting to transform wind energy into electricity. Erturk et al [3] performed an experimental study on generating electricity from thin curved airfoils with macro-fiber composite (MFC) piezoceramics under airflow excitation. De Marqui et al [4] studied the piezoaeroelastic problem of energy harvesting with respect to the airflow excitation of a cantilevered plate with embedded piezoceramics. Tang et al [5] proposed a flexible energy harvester inspired by flapping flag dynamics. Their mechanism used self-excited vibrations derived from the axial flow over a cantilever. Pobering et al [6] studied energy harvesting experimentally and numerically by using vortexinduced oscillations of piezoelectric cantilevers located behind bluff bodies. Bryant and Garcia [7] proposed and tested a novel energy harvester by using the aeroelastic
This is an Open Access article distributed under the terms of the Creative Commons Attribution Non-Commercial License (http://creativecommons.org/licenses/by$\mathrm{nc} / 3.0 /$ which permits unrestricted non-commercial use, distribution, and reproduction in any medium, provided the original work is properly cited. (c) * Professor, Corresponding author: jsbae@kau.ac.kr ** Professor 
flutter oscillation of a piezoelectric beam with a simple flap attached to the beam tip. Bryant et al [8] examined the sensitivity of driving aeroelastic instability to several design parameters. Dunnmon et al [9] performed theoretical modeling by augmenting a system of nonlinear equations for an electroelastic beam with a vortex-lattice potential flow model and discussed their method by comparing existing relevant aerodynamic models. Sousa et al [10] performed the linear and nonlinear modeling of a 2-DOF piezoaeroelastic airfoil and exploited the combined nonlinearities with free play and cubic hardening in wind energy harvesting. De Melo Anicézio et al [11] extended the previous work [10] to electromagnetic energy harvesting and investigated the problems of linear and nonlinear aeroelastic energy harvesting using electromagnetic induction. Abdelkefi and Hajj [12] investigated the effects of freeplay nonlinearity on a wing-based piezoaeroelastic energy harvesting system. Abdelkefi and Nuhait [13] investigated the effects of unsteady aerodynamics on the performance of wing-based piezoaeroelastic energy harvesters.

Although some research efforts have been directed toward energy harvesting using linear and nonlinear aeroelastic phenomena, piezo-aeroelastic energy harvesting has not yet been fully examined in a design context. Linear aeroelastic responses include convergent oscillation at air speeds below flutter speed, neutrally stable oscillation at flutter speed, and divergent oscillation over flutter speed. Flutter, which is a divergent oscillation, happens catastrophically in the vicinity of flutter speed and causes a structural failure. Hence, energy harvesting using a linear form of aeroelastic oscillation such as flutter might be very dangerous or impossible. More recently, Bae and Inman [14] investigated the linear and nonlinear aeroelastic characteristics of a piezo-aeroelastic energy harvester. They introduced several new nondimensional parameters and presented nondimensional piezo-aeroelastic equations to exploit the effects of parameters on the piezo-aeroelastic characteristics. Based on their results of linear and nonlinear piezo-aeroelastic energy harvesting, they discussed how to use nonlinear aeroelastic characteristics for energy harvesting.

Nonlinear aeroelastic responses include convergent oscillation, limit cycle oscillation (LCO), periodic motion, chaotic motion, and divergent oscillation [15, 16]. Among these nonlinear aeroelastic responses, LCOs can be observed at air speeds below or over the linear flutter speed dependent on aeroelastic system parameters such as natural frequencies, natural modes, and structural nonlinearity types. Conner et al [17] performed a numerical and experimental study on the nonlinear behavior of a two-dimensional airfoil section with control surface free play, and they observed LCOs. Tang et al [18] presented the theoretical model of a three-degrees-of-freedom (3DOF) aeroelastic model with freeplay using a reduced order model. Their model was verified through a comparison with experimental data. Bae et al [16] investigated the linear and nonlinear aeroelastic characteristics of a wing with a control surface. They demonstrated that various types of LCOs, periodic motions, and chaotic motions could be observed in a wide range of air speeds below the linear flutter speed. They also observed an LCO amplitude jump due to a change in the LCO mode.

In the present study, the authors propose a piezoaeroelastic energy harvester using a nonlinear trailing-edge flap (TEF) and perform a preliminary study of the concept. Fig. 1 shows the schematic of the piezo-aeroelastic energy harvester with the nonlinear TEF. Due to the presence of the nonlinear TEF, LCOs are observed in a wide range of air speeds below the linear flutter speed. These LCOs can be used to harvest energy without serious structural damage of the wing. The authors extend their previous work [14] to a two-dimensional airfoil section with a nonlinear TEF to investigate its nonlinear aeroelastic characteristics. Fig. 2 shows a typical piezo-aeroelastic section with a TEF. The piezoelectric coupling is introduced through a plunge motion, while freeplay nonlinearity is introduced to the flap motion. The root-locus (RL) method [19] and time-integration method $[19,20]$ are used to carry out the frequency domain analysis and time domain analysis, respectively. The linearizing method describing function(DF) [19] is used for the frequency domain analysis of a nonlinear aeroelastic system. The aeroelastic results predicted by the present method are verified against the experimental results [17]. From the linear and nonlinear piezo-aeroelastic analyses, the LCO characteristics of the proposed energy harvester with the nonlinear TEF are investigated in both the frequency and time domains. Finally, the authors discuss the air speed range for effective

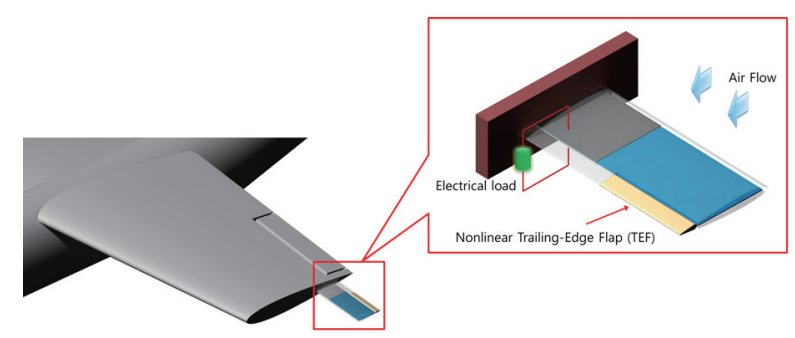

Fig. 1. Schematic of a piezo-aeroelastic energy harvester with a nonlinear TEF 
piezo-aeroelastic energy harvesting.

\section{Theoretical Analysis}

\subsection{Piezo-aeroelastic modeling of a 2-D typical section model with a trailing-edge flap}

Figure 2 shows a piezo-aeroelastic energy harvester model of a 2-DOF typical section with a trailing-edge flap (TEF). The equations of the motion of the energy harvester, as shown in Fig. 1, are

$$
\begin{aligned}
& \left(m+m_{f}\right) \ddot{h}+S_{\alpha} \ddot{\alpha}+S_{\delta} \ddot{\delta}+k_{h} h-k_{h} D v=-L(t) \\
& S_{\alpha} \ddot{h}+I_{\alpha} \ddot{\alpha}+\left[I_{\delta}+S_{\delta} b(c-a)\right] \ddot{\delta}+K_{\alpha} \alpha=M_{\alpha}(t) \\
& S_{\delta} \ddot{h}+\left[I_{\delta}+S_{\delta} b(c-a)\right] \ddot{\alpha}+I_{\delta} \ddot{\delta}+K_{\delta} \delta=M_{\delta}(t) \\
& C_{p} \dot{v}+\frac{1}{R_{e q}} v+k_{h} D \dot{h}=0
\end{aligned}
$$

where

$$
\begin{aligned}
& m=\int \rho d \bar{x} \\
& S_{\alpha}=\int \rho \bar{x} d \bar{x} \quad S_{\delta}=\int \rho \bar{x}_{1} d \bar{x}_{1} \\
& I_{\alpha}=\int \rho \bar{x}^{2} d \bar{x} \quad I_{\delta}=\int \rho \bar{x}_{1}^{2} d \bar{x}_{1} \\
& \bar{x}=x-e=x-b(1+a) \\
& \bar{x}_{1}=x-b(1+c) \\
& m_{f}: \text { fixture mass } \\
& \rho: \text { air density } \\
& \mathrm{U}_{\infty}: \text { air speed } \\
& c: \text { chord length of airfoil } \\
& C_{l}: 2 \text {-D lift coefficient } \\
& C_{m}: 2 \text {-D moment coefficient }
\end{aligned}
$$

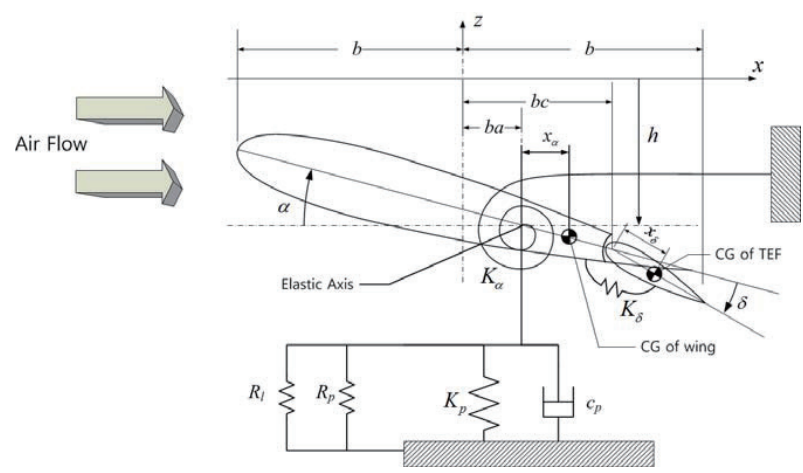

Fig. 2. Piezo-aeroelastic model of a 2-D typical section with a TEF

$$
\begin{aligned}
& D \text { : electromechanical coupling } \\
& C_{p} \text { : capacitance of piezoelectric material } \\
& R_{e q}: \text { resistance of electric circuit } \\
& L(t), M(t): \text { lift and moment }
\end{aligned}
$$

To nondimensionalize the equations of motion, the following nondimensional parameters are introduced.

$$
\begin{aligned}
& \xi=\frac{h}{b}, \eta=\frac{D v}{b}, \tau=\frac{U_{\infty} t}{b}, x_{\alpha}=\frac{s_{\alpha}}{m b}, x_{\delta}=\frac{s_{\delta}}{m b}, \\
& r_{\alpha}=\sqrt{\frac{I_{\alpha}}{m b^{2}}}, \bar{\omega}_{\alpha}=\frac{\omega_{\alpha}}{\omega_{h}}, \bar{\omega}_{\delta}=\frac{\omega_{\delta}}{\omega_{h}}, \omega_{\alpha}=\sqrt{\frac{K_{\alpha}}{I_{\alpha}}}, \\
& \omega_{\delta}=\sqrt{\frac{K_{\delta}}{I_{\delta}}}, \omega_{h}=\sqrt{\frac{K_{h}}{m}}, \underline{U}=\frac{U_{\infty}}{b \omega_{h}}, \beta=\frac{m+m_{f}}{m}
\end{aligned}
$$

Using these parameters, the nondimensional form of Equations (1)-(4) can be written as

$$
\begin{aligned}
& \beta \ddot{\xi}+x_{\alpha} \ddot{\alpha}+x_{\delta} \ddot{\delta}+\frac{1}{\underline{U}^{2}} \xi-\frac{1}{\bar{U}^{2}} \eta=-\frac{b}{m U_{\infty}^{2}} L \\
& \frac{x_{\alpha}}{r_{\alpha}^{2}} \ddot{\xi}+\ddot{\alpha}+\left[\frac{r_{\delta}^{2}}{r_{\alpha}^{2}}+\frac{x_{\delta}}{r_{\alpha}^{2}}(c-a)\right] \ddot{\delta}+\frac{\bar{\omega}_{\alpha}^{2}}{\underline{U}^{2}} \alpha=\frac{b^{2}}{I_{\alpha} U_{\infty}^{2}} M_{\alpha} \\
& \frac{x_{\delta}}{r_{\delta}^{2}} \ddot{\xi}+\left[1+\frac{x_{\delta}}{r_{\delta}^{2}}(c-a)\right] \ddot{\alpha}+\ddot{\delta}+\frac{\bar{\omega}_{\delta}^{2}}{\underline{U}^{2}} \delta=\frac{b^{2}}{I_{\delta} U_{\infty}^{2}} M_{\delta} \\
& \bar{C}_{p} \dot{\eta}+\frac{1}{\underline{U} \underline{R}_{e q}} \eta+\dot{\xi}=0
\end{aligned}
$$

where nondimensionalized resistance and capacitance are $\underline{R}_{e q}=m D^{2} \omega_{h}^{3} R_{e q}\left(R_{e q} \approx R_{l}\right)$ and $\underline{C}_{e q}=C_{p} / m D^{2} \omega_{h^{\prime}}^{2}$ respectively.

The vector forms of Equations (4) and (8) are

$$
[M]\left\{\begin{array}{c}
\ddot{\xi} \\
\ddot{\alpha} \\
\ddot{\delta}
\end{array}\right\}+[C]\left\{\begin{array}{c}
\dot{\xi} \\
\dot{\alpha} \\
\dot{\delta}
\end{array}\right\}+[K]\left\{\begin{array}{l}
\xi \\
\alpha \\
\delta \\
\eta
\end{array}\right\}=\{F\}
$$

where $[C]$ is the structural damping matrix and

$$
\begin{aligned}
& {[M]=\left[\begin{array}{ccc}
\beta & x_{\alpha} & x_{\delta} \\
\frac{x_{\alpha}}{r_{\alpha}^{2}} & 1 & \frac{r_{\delta}^{2}}{r_{\alpha}^{2}}+\frac{x_{\delta}}{r_{\alpha}^{2}}(c-a) \\
\frac{x_{\delta}}{r_{\delta}^{2}} & 1+\frac{x_{\delta}}{r_{\delta}^{2}}(c-a) & 1
\end{array}\right]} \\
& {[C]=\left[\begin{array}{ccc}
\frac{2 \zeta_{h}}{\underline{U}} & 0 & 0 \\
0 & \frac{2 \zeta_{\alpha} \bar{\omega}_{\alpha}}{\underline{U}} & 0 \\
0 & 0 & \frac{2 \zeta_{\delta} \bar{\omega}_{\delta}}{\underline{U}}
\end{array}\right]} \\
& {[K]=\left[\begin{array}{cccc}
\frac{1}{\underline{U}^{2}} & 0 & 0 & -\frac{1}{\underline{U}^{2}} \\
0 & \frac{\bar{\omega}_{\alpha}^{2}}{\underline{U}^{2}} & 0 & 0 \\
0 & 0 & \frac{\bar{\omega}_{\delta}^{2}}{\underline{U}^{2}} & 0
\end{array}\right],\{F\}=\left\{\begin{array}{l}
-\bar{L} \\
\bar{M}_{\alpha} \\
\bar{M}_{\delta}
\end{array}\right\}=\left\{\begin{array}{l}
-\frac{b}{m U_{\infty}^{2}} L \\
\frac{b^{2}}{I_{\alpha} U_{\infty}^{2}} M_{\alpha} \\
\frac{b^{2}}{I_{\delta} U_{\infty}^{2}} M_{\delta}
\end{array}\right\}}
\end{aligned}
$$




\subsection{Aerodynamic influence coefficients and ap- proximations}

The aerodynamic force terms in Equation (9) can be expressed by using aerodynamic influence coefficients $[Q(k)]$ as follows.

$$
\{F(\tau)\}=[Q(k)]\{u(\tau)\}
$$

where $k\left(=\frac{\omega b}{U_{\infty}}\right)$ is the reduced frequency.

The aerodynamic influence coefficients are generally calculated for the tabulated reduced frequencies by using various unsteady aerodynamic theories such as the Theodorsen function [20], panel method, and CFD. Theodorsen's unsteady lift and moments of a thin airfoil with a trailing-edge flap can be written as follows.

$$
\begin{aligned}
L= & \rho b^{2}\left(\pi U_{\infty} \dot{\alpha}+\pi \ddot{h}-\pi b a \ddot{\alpha}-U_{\infty} T_{4} \dot{\delta}-T_{1} b \ddot{\delta}\right) \\
& +2 \pi \rho b U_{\infty} C(k)\left[U_{\infty} \alpha+\dot{h}+b\left(\frac{1}{2}-a\right) \dot{\alpha}+\frac{1}{\pi} T_{10} U_{\infty} \delta\right. \\
& \left.+\frac{1}{2 \pi} b T_{11} \dot{\delta}\right] \\
M_{\alpha}= & -\rho b^{2}\left\{\pi U_{\infty} b\left(\frac{1}{2}-a\right) \dot{\alpha}+\pi b^{2}\left(\frac{1}{8}+a^{2}\right) \ddot{\alpha}+\left(T_{4}+T_{10}\right) U_{\infty}^{2} \delta\right. \\
& \left.+\left(T_{1}-T_{8}-(c-a) T_{4}+\frac{1}{2} T_{11}\right) U_{\infty} b \dot{\delta}-\left[T_{7}+(1-a) T_{1}\right] b^{2} \ddot{\delta}-\pi a b \ddot{h}\right\} \\
& +2 \pi \rho b^{2} U_{\infty}\left(a+\frac{1}{2}\right) C(k)\left[U_{\infty} \alpha+\dot{h}+b\left(\frac{1}{2}-a\right) \dot{\alpha}+\frac{1}{\pi} U_{\infty} T_{10} \delta+\frac{1}{2 \pi} b T_{11} \dot{\delta}\right] \\
M_{\delta}= & -\rho b^{2}\left\{\left[-2 T_{9}-T_{1}+T_{4}\left(a-\frac{1}{2}\right)\right] b U_{\infty} \dot{\alpha}+2 T_{13} b^{2} \ddot{\alpha}\right. \\
& \left.+\frac{1}{\pi}\left(T_{5}-T_{4} T_{10}\right) U_{\infty}^{2} \delta-\frac{1}{2 \pi} T_{4} T_{11} b U_{\infty} \dot{\delta}-\frac{1}{\pi} T_{3} b^{2} \ddot{\delta}-T_{1} b \ddot{h}\right\} \\
& -\rho b^{2} U_{\infty} T_{12} C(k)\left[U_{\infty} \alpha+\dot{h}+b\left(\frac{1}{2}-a\right) \dot{\alpha}+\frac{1}{\pi} T_{10} U_{\infty} \delta+\frac{1}{2 \pi} b T_{11} \dot{\delta}\right]
\end{aligned}
$$

The new variables $\left(T_{i}\right)$ are presented in Appendix A. $C(k)$ is a function of Henkel's functions [21]. The components of the nondimensional AICs are also presented in Appendix B.

For the time-domain analysis, the AICs must be calculated for arbitrary oscillation. Hence, the AICs calculated for the tabulated reduced frequencies must be approximated by continuous functions. In this study, Karpel's minimumstate approximation (MSA) [22] is used. The AICs [Q(k)] approximated by Karpel's MSA are

$$
[Q(j k)]=\left[P_{1}\right] \bar{s}^{2}+\left[P_{2}\right] \bar{s}+\left[P_{3}\right]+[\bar{D}](\bar{s}[I]-[\bar{R}])^{-1}[\bar{E}] \bar{s}
$$

where $\bar{s}=\frac{b}{U_{\infty}} s=j k$.

\subsection{State-space aeroelastic analysis method}

Substituting Equation (14) into the Laplace transform of
Equation (9) leads to the following state-space equations.

$$
\left\{\begin{array}{c}
\ddot{\xi} \\
\ddot{\alpha} \\
\ddot{\delta}
\end{array}\right\}=-[\bar{M}]^{-1}[\bar{C}]\left\{\begin{array}{c}
\dot{\xi} \\
\dot{\alpha} \\
\dot{\delta}
\end{array}\right\}-[\bar{M}]^{-1}[\bar{K}]\left\{\begin{array}{c}
\xi \\
\alpha \\
\delta \\
\eta
\end{array}\right\}+[\bar{M}]^{-1}[\bar{D}]\left\{X_{a}(\bar{s})\right\}
$$

where

$$
[\bar{M}]=[M]-\left[P_{1}\right],[\bar{C}]=[C]-\left[P_{2}\right],[\bar{K}]=[K]-\left[P_{3}\right]
$$

The aerodynamic states in Equation (15) are defined by

$$
\left\{X_{a}(\bar{s})\right\}=(\bar{s}[I]-[\bar{R}])^{-1}[\bar{E}] \bar{s}\{X(\bar{s})\}
$$

Equation (8) can be rearranged as

$$
\dot{\eta}=-\frac{1}{\underline{C}_{p}} \dot{\xi}-\frac{1}{\underline{U} \underline{C}_{p} \underline{R}_{e q}} \eta
$$

Defining the new state variable as $\mathrm{z}=\left[\begin{array}{llll}\dot{\xi} & \dot{\alpha} & \dot{\delta} & \dot{\delta}\end{array}\right]^{T}$ and rearranging Equations (15)-(17) leads to the following statesate piezo-aeroelastic equations.

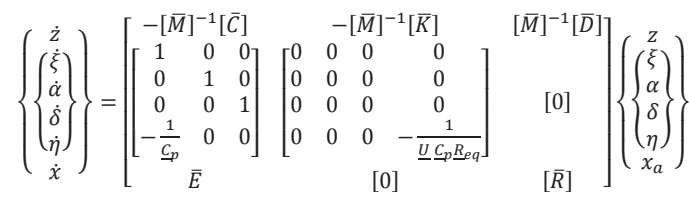

There exist two approaches to obtain the solutions for Equation (18) $[14,19]$. One is the RL method in the frequency domain, and the other is the time-integration method in the time domain. The RL method is used to solve the eigenvalue problem in Equation (18). As the air speed increases, the eigenvalues are calculated. When the real part of the eigenvalues becomes positive, flutter occurs. The time-integration method is used to integrate Equation (18) numerically and to calculate the time response for a specific air speed using a numerical integration method such as the Runge-Kutta method.

\subsection{Structural nonlinearity}

When structural nonlinearity exists in the flap motion, Equation (9) can be written as

$$
[M]\left\{\begin{array}{c}
\ddot{\xi} \\
\ddot{\alpha} \\
\ddot{\delta}
\end{array}\right\}+[C]\left\{\begin{array}{c}
\dot{\xi} \\
\dot{\alpha} \\
\dot{\delta}
\end{array}\right\}+[K]_{\text {linear }}\left\{\begin{array}{c}
\xi \\
\alpha \\
\delta \\
\eta
\end{array}\right\}=\{F\}-\{f\}_{\text {nonlinear }}
$$

where $[K]_{\text {linear }}$ and $\{f\}_{\text {nonlinear }}$ are the linear stiffness matrix and nonlinear restoring force vector, respectively, as follows.

$$
[K]_{\text {linear }}=\left[\begin{array}{cccc}
\frac{1}{\underline{U}^{2}} & 0 & 0 & -\frac{1}{\underline{U}^{2}} \\
0 & \frac{\bar{\omega}_{\alpha}^{2}}{\underline{U}^{2}} & 0 & 0 \\
0 & 0 & 0 & 0
\end{array}\right]
$$




$$
\{f\}_{\text {nonlinear }}=\left\{\begin{array}{c}
0 \\
0 \\
f(\delta)
\end{array}\right\}
$$

If the nonlinearity is freeplay, Equation (21) can be written as

$$
f(\delta)=\left\{\begin{array}{cc}
K_{\delta}(\delta-s) & (\delta>s) \\
0 & (-s<\delta<s) \\
K_{\delta}(\delta+s) & (\delta<-s)
\end{array}\right.
$$

where $s$ is the freeplay.

The equivalent stiffness of a nonlinear spring in Equation (22) is required to use the RL method and can be obtained by using the describing function method as follows.

$$
K_{e q}(\delta)=\left\{\begin{array}{cc}
0 & (0<\bar{\delta}<s) \\
\frac{K_{\delta}}{\pi}\left[\pi-2 \sin ^{-1} \frac{s}{\bar{\delta}}-\sin \left(2 \sin ^{-1} \frac{s}{\bar{\delta}}\right)\right] & (\bar{\delta}>s)
\end{array}\right.
$$

where $\bar{\delta}$ is the amplitude of pitch angle $\delta$.

Substituting Equation (23) into Equation (3), the stiffness matrix in Equation (20) can be obtained by

$$
[K]_{\text {linear }}=\left[\begin{array}{cccc}
\frac{1}{\underline{U}^{2}} & 0 & 0 & -\frac{1}{\underline{U}^{2}} \\
0 & \frac{\bar{\omega}_{\alpha}^{2}}{\underline{U}^{2}} & 0 & 0 \\
0 & 0 & \frac{\bar{\omega}^{2}(\delta)}{\underline{U}^{2}} & 0
\end{array}\right]
$$

where

$$
\bar{\omega}^{2}(\delta)=\bar{\omega}^{2}\left\{\begin{array}{cc}
0 & (0<\bar{\delta}<s) \\
\frac{1}{\pi}\left[\pi-2 \sin ^{-1} \frac{s}{\bar{\delta}}-\sin \left(2 \sin ^{-1} \frac{s}{\bar{\delta}}\right)\right] & (\bar{\delta}>s)
\end{array}\right.
$$

For the time-domain analysis, substituting Equations (20) and (22) into Equation (19) leads to the following nonlinear state-space piezo-aeroelastic equations.

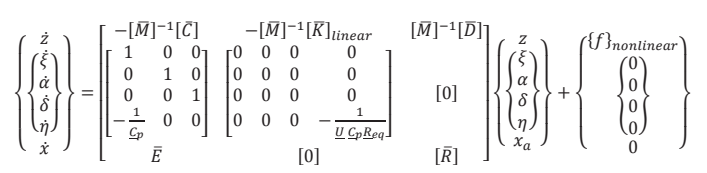

The nonlinear time response can be obtained by integrating Equation (26).

\section{Results and Discussion}

\subsection{Verifications of present analysis}

To verify the accuracy of the present aeroelastic analysis, the present results of RL method are compared to the predicted and experimental data of Reference [17]. Table 1 shows a comparison of the flutter speeds and flutter frequencies of a 2-D airfoil with a TEF. The present results are in good agreement with those of the prediction in Reference [17], while both are a little different from the experimental data.

Table 2 shows the comparison of the flutter speeds and flutter frequencies of a 2-D piezo-aeroelastic energy harvester. The present results are in good agreement with those of the experiments and predictions in Erturk and Inman [2]. The flutter speed for the shot-circuit $\left(R_{l} \approx 0\right)$ is predicted in the present study using the RL method to be $8.936 \mathrm{~m} / \mathrm{s}$, overestimating the experimental value of 8.85 $\mathrm{m} / \mathrm{s}$ by $0.96 \%$. The present study predicts the flutter speed to be $8.975 \mathrm{~m} / \mathrm{s}$ for the open-circuit $\left(R_{l} \approx \infty\right)$, overestimating the experimental value of $8.90 \mathrm{~m} / \mathrm{s}$ by $0.84 \%$.

\subsection{Linear aeroelastic characteristics of a piezo- aeroelastic energy harvester with a TEF}

Understanding the linear piezo-aeroelastic characteristics

Table 1. Verification of present results

\begin{tabular}{cccccc}
\hline & \multicolumn{2}{c}{ Present } & \multicolumn{2}{c}{ Reference [17] } \\
\hline & unit & V-g(Bae 2002) & Root-Locus & Numerical & Experiments \\
\hline Flutter speed & $\mathrm{m} / \mathrm{s}$ & 23.96 & 24.06 & 23.9 & 20.6 \\
\hline \multirow{2}{*}{ Flutter frequency } & $\mathrm{Hz}$ & 6.22 & 6.11 & 6.112 & 5.47 \\
\hline
\end{tabular}

Table 2. Comparison of piezo-aeroelastic flutter speeds and frequencies

\begin{tabular}{clllll}
\hline Resistance $(\Omega)$ & $U_{f}(\mathrm{~m} / \mathrm{s})$ & $U_{f}(\mathrm{~m} / \mathrm{s})$ & $\omega_{f}(\mathrm{~Hz})$ & $U_{f}(\mathrm{~m} / \mathrm{s})$ & $\omega_{f}(\mathrm{~Hz})$ \\
$\approx 0$ (Short Circuit) & 8.85 & & & 8.936 & 5.167 \\
100 & 8.85 & 9.06 & 5.17 & 8.936 & 5.167 \\
$100 \mathrm{k}$ & 9.30 & 9.56 & 5.14 & 9.346 & 5.147 \\
$\approx \infty$ (Open Circuit) & 8.90 & & & 8.975 & 5.187 \\
\hline
\end{tabular}


of an energy harvester with a TEF is important when investigating its nonlinear characteristics. Table 3 shows the parameters used in the present analysis. The flutter speed and frequency variation of the linear piezo-aeroelastic energy harvester is shown in Fig. 3. Fig. 3 shows that the flutter speed and frequency vary considerably for various frequency ratios $\left(\bar{\omega}_{\delta}\right)$, and there exist frequency ratio values at which the abrupt change in flutter speed and frequency occurs. This is due to the change in flutter modes. Three kinds of flutter modes, Type A, Type B, and Type C, exist in Fig. 3. Type A is a plunge-flap mode flutter in which the frequency ratio is less than about 0.7 . Type $B$ is a pitch-flap mode flutter in which the frequency ratio is between about 0.7 and 1.3. Type $\mathrm{C}$ is a conventional plunge-pitch mode flutter in which the frequency ratio is larger than about 1.3. Bae et al [15] characterized four regions according to the flutter mode shapes of an aircraft wing with a control surface. The present Type A, Type B, and Type C modes are similar to Bae et al's Region IV, Region I, and Region III, respectively. Their study showed that Region IV was very important in terms of the nonlinear aeroelastic characteristics, while it may not have been important in terms of the linear characteristics. Fig. 4 shows the effect of resistance on the linear piezo-aeroelastic characteristics. When $\underline{R}_{e q}$ is about 0.025 , the flutter speed reaches its maximum.

Table 3. Parameters used in the present study

\begin{tabular}{cc}
\hline Parameters & Values \\
\hline$x_{\alpha}$ & 0.260 \\
\hline$x_{\delta}$ & 0.0124 \\
\hline$r_{\alpha}^{2}$ & 0.504 \\
\hline$r_{\delta}^{2}$ & 0.0062 \\
\hline$\mu\left(=\frac{m}{\pi \rho b^{2} l}\right)$ & 29.6 \\
\hline$a$ & -0.5 \\
\hline$c$ & 0.5 \\
\hline$\beta$ & 2.597 \\
\hline$C_{p}$ & 40.02 \\
\hline $\bar{C}^{2} \omega_{h}^{2}$ & 1.3 \\
\hline $\bar{\omega}_{\alpha}$ & 1.8 \\
\hline $\bar{\omega}_{\delta}$ & 0.125 \\
\hline$b$ & 0.5 \\
\hline$l$ & 0.0035 \\
\hline$\varsigma_{h}$ & 0.06 \\
\hline$\varsigma_{\alpha}$ & 0.02 \\
\hline
\end{tabular}

\subsection{Nonlinear aeroelastic characteristics of a piezo- aeroelastic energy harvester with a TEF}

In the present study, the stiffness of the flap motion is assumed to have freeplay, as described by Equation (22). The equivalent stiffness of Equation (23) is used for the frequency-domain nonlinear analysis by using the RL method. Fig. 5 shows the LCO characteristics of the piezoaeroelastic energy harvester with a nonlinear TEF when $\underline{C}_{p}$ $=40.02$ and $\underline{R}_{e q}=0.02$. The results of the frequency domain analysis and time domain analysis agree well, while some LCOs cannot be observed in the frequency domain analysis. For the linear aeroelastic system, the piezo-aeroelastic response dissipates due to aerodynamic damping at air speeds below the linear flutter speed $\left(\underline{U}_{F}=4.77\right)$. However, as shown in Fig. 5, various LCO types can be observed at air speeds below the linear flutter speed, and an abrupt change of LCO amplitude is observed. This amplitude change is due to the change in LCO flutter mode.

Figures 6 - 8 show the LCO amplitudes for the plunge, pitch, and flap motions. Two LCO types are observed at air

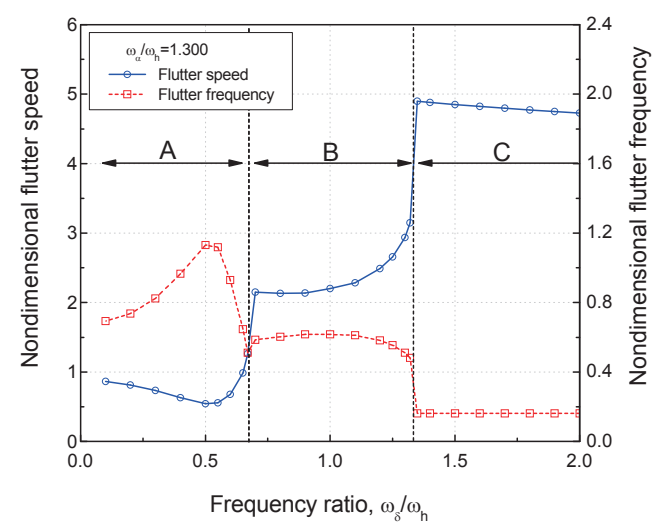

Fig. 3. Linear piezo-aeroelastic characteristics for various frequency ratios $\left(\bar{\omega}_{\delta}\right)\left(\underline{C}_{p}=40.02, \underline{R}_{e q}=0.02\right)$

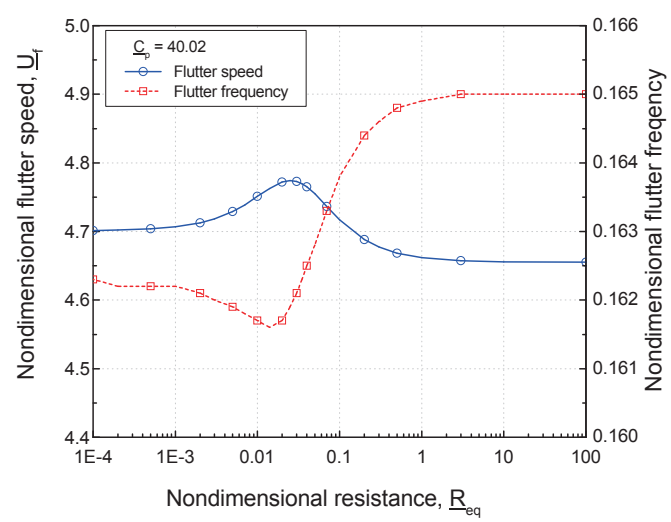

Fig. 4. Effects of $\underline{R}_{e q}$ on flutter speed and frequency $\left(\bar{\omega}_{\delta}=1.3, \omega \bar{\omega}_{\delta}\right.$ $=1.8)$ 
speeds below the linear flutter speed. One LCO is observed at air speeds from 0.9 to 2.4. The other LCO is observed at air speeds from 2.2 to 4.6 . At air speeds from 2.2 to 2.4 , both LCOs are observed dependent on initial conditions. These LCOs have different LCO modes. The LCOs observed from 0.9 to 2.4 are the Type A plunge-flap mode flutters, as shown in Fig. 3. Fig. 9 shows the time histories of the plunge, pitch, and flap motions when the air speed $(\underline{U})$ and initial condition $\left(\delta_{0} / s\right)$ are both 1.5 . The amplitude of the plunge motion is relatively large. The LCOs observed from 2.2 to 4.6 are Type B pitch-flap mode flutters. The plunge amplitude of these LCOs is relatively small. At air

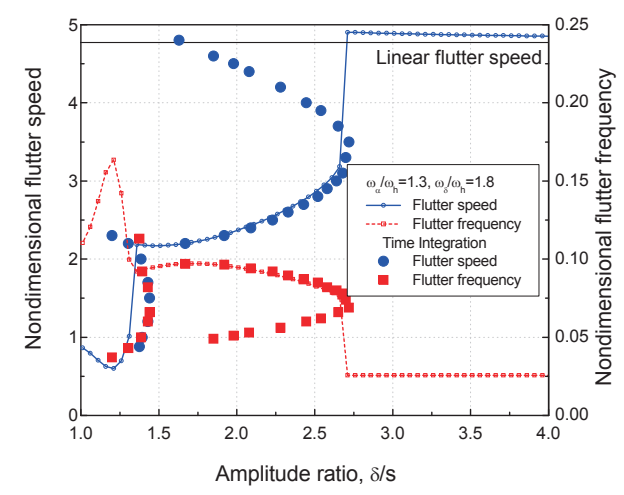

Fig. 5. LCO boundary of piezo-aeroelastic energy harvester $\left(\underline{C}_{p}=40.02, \underline{R}_{e q}=0.02\right)$

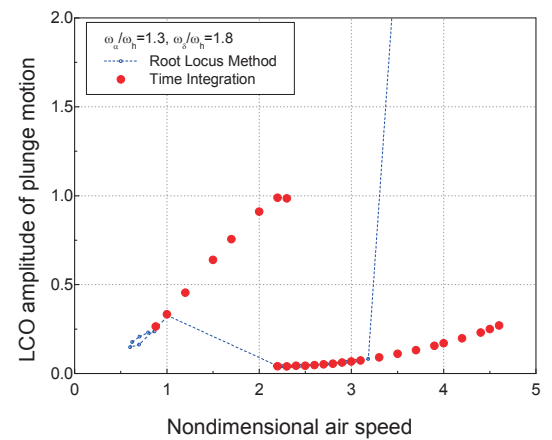

Fig. 6. LCO amplitude of plunge motion

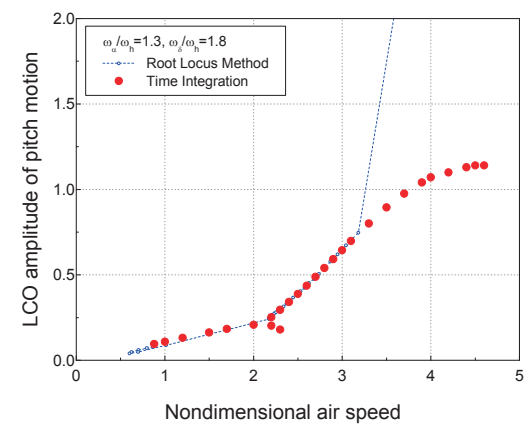

Fig. 7. LCO amplitude of pitch motion

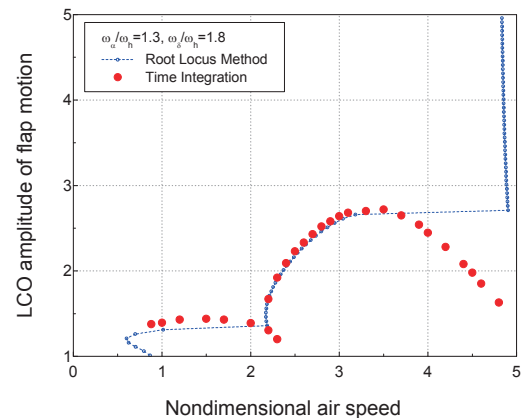

Fig. 8. LCO amplitude of flap motion
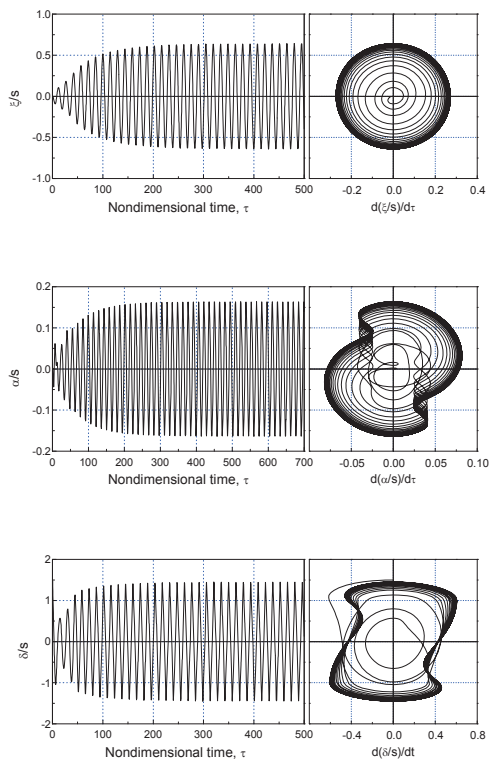

Fig. 9. Time histories when $\underline{U}=1.5$ and $\delta_{0} / \mathrm{s}=1.5$
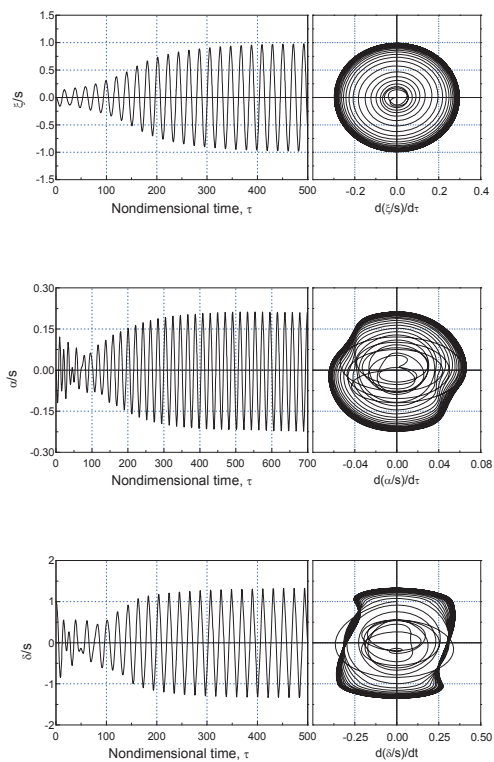

Fig. 10. Time histories when $\underline{U}=2.2$ and $\delta_{0} / \mathrm{s}=1.0$ 
speeds from 2.2 to 2.4 in particular, both Type A and Type $\mathrm{B}$ are observed. Figures 10 and 11 show the time histories when the air speed $(\underline{U})$ is 2.2 and initial conditions are 1.0 and 3.0, respectively. When the initial condition $\left(\delta_{0} / s\right)$ is 1.0 , the Type A LCO remains, while when $\delta_{0} / s$ is 3.0, the piezo-aeroelastic response goes to the Type B LCO. Fig. 12 shows the time histories when $\underline{U}$ is 4.0 and a Type B LCO is observed independent of the initial condition. Bae et al [15] shows how these Type A and Type B LCOs oscillate
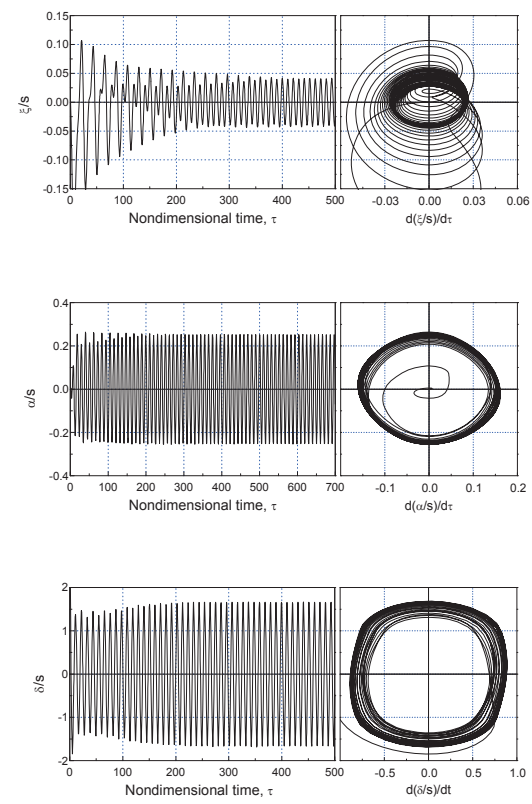

Fig. 11. Time histories when $\underline{U}=2.2$ and $\delta_{0} / \mathrm{s}=3.0$
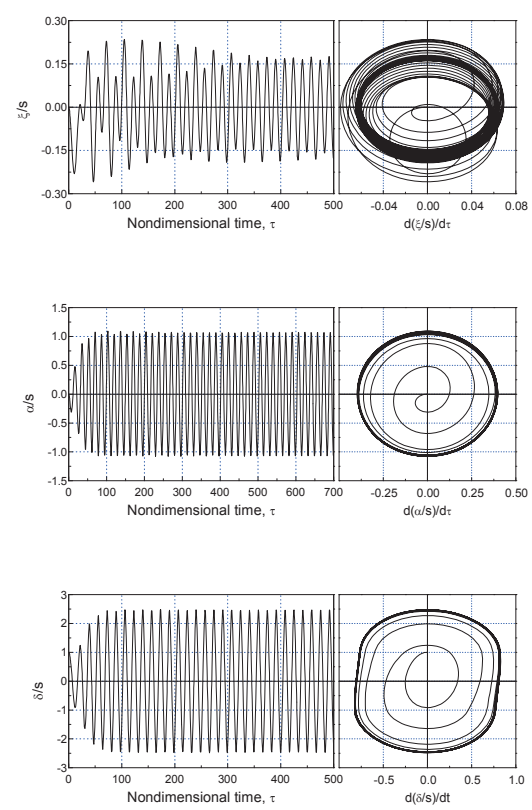

Fig. 12. Time histories when $\underline{U}=4.0$ and $\delta_{0} / \mathrm{s}=1.0$
(Region IV and Region I in Fig. 6, Bae et al [15]). Figs. 13 and 14 show the time histories at an air speed of 5.2, over the linear flutter speed. Dependent on the initial condition, periodic motions are observed or piezo-aeroelastic responses become divergent. Hence, piezo-aeroelastic energy harvesting is impossible in the vicinity of the linear flutter speed.

LCO amplitude of plunge motion is important for the proposed energy harvester. The plunge amplitudes of LCOs
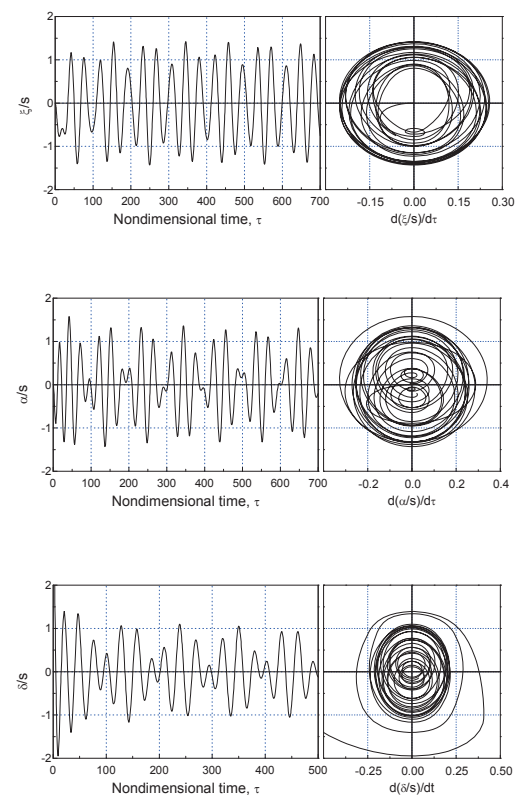

Fig. 13. Time histories when $\underline{U}=5.2$ and $\delta_{0} / \mathrm{s}=4.0$
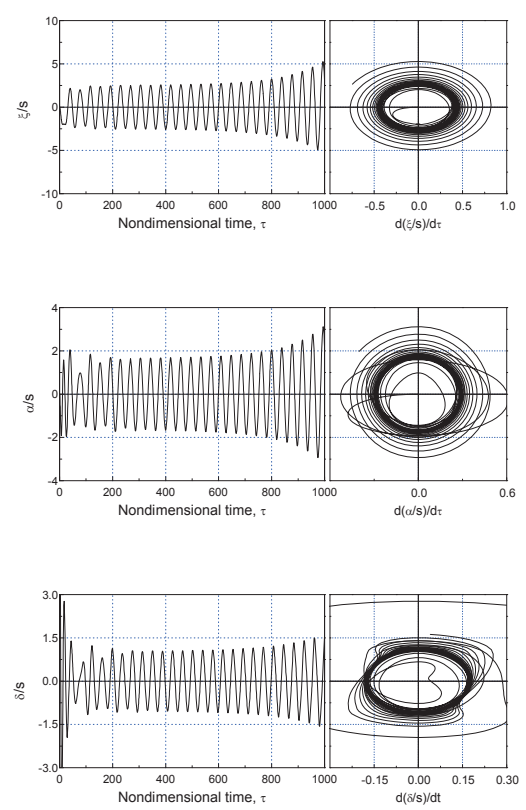

Fig. 14. Time histories when $\underline{U}=5.2$ and $\delta_{0} / \mathrm{s}=10.5$ 
observed at air speed through 0.9 to 2.2 are relatively large as shown in Fig. 6. Hence, these Type A LCOs can be used effectively for the proposed energy harvester at air speeds from 0.9 to 2.2. Fig. 15 shows the voltage amplitude of the proposed energy harvester. At air speeds from 0.9 to 2.2, the voltage amplitude is significantly large. Also, this air speed range is definitely safe with respect to aeroelastic instability.

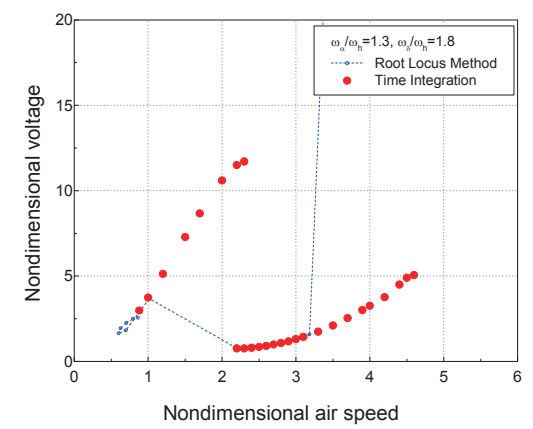

Fig. 15. LCO amplitude of voltage

\section{Conclusions}

In the present study, the linear and nonlinear aeroelastic characteristics of a piezo-aeroelastic energy harvester with a nonlinear trailing-edge flap (TEF) are investigated. The linear and nonlinear piezo-aeroelastic modelings of a simple, 3-DOF typical section are presented. A RL and a time-integration method are used to carry out the frequency domain analysis and time domain analysis, respectively. The results of these methods are verified against previously published experimental results.

As mentioned in the previous section, the aeroelastic responses dissipate due to the presence of aerodynamic damping at air speeds below the flutter speed. Flutter, which is an aeroelastic instability, occurs catastrophically in the vicinity of flutter speed. Hence the use of a linear aeroelastic response for energy harvesting might be very dangerous or impossible. The aeroelastic systems have several types of structural nonlinearities such as freeplay and cubic hardening. These nonlinearities can increase or decrease the nonlinear flutter boundary. For freeplay when the frequency ratio is larger than 1.0, stable LCOs with various amplitudes occur at a wide range of air speeds below the flutter speed, and these LCOs can be used for energy harvesting. When the frequency ratio is smaller than 1.0, unstable LCOs are observed at air speeds over the flutter speed, and these LCOs may not be used for energy harvesting. For cubic hardening when the frequency ratio is larger than 1.0, stable LCOs occur at air speeds over the flutter speed, and these LCOs can be used for energy harvesting. Generally, an aeroelastic system is designed to avoid flutter and possess safety margins because flutter causes catastrophic failure. LCOs at air speeds over the flutter speed cannot be used for energy harvesting even though they are stable. Therefore, stable LCOs below the linear flutter are an excellent source for energy harvesting without aeroelastic instability.

\section{Acknowledgements}

This work was supported by the Korea Institute of Energy Technology Evaluation (2014303021130). This support is gratefully acknowledged. The second author gratefully acknowledges the support in part provided by the US Air Force Office of Scientific Research under the grant number FA9550-14-1-0246 "Electronic Damping in Multifunctional Material Systems" monitored by Dr BL Lee.

\section{References}

[1] Anton, S.R., and Sodano, H.A., "A Review of Power Harvesting using Piezoelectric Materials (2003-2006)," Smart Materials and Structures, Vol. 16, 2007, R1-R21.

[2] Erturk, A. and Inman, D. J. Piezoelectric Energy Harvesting, Wiley, New York, 2011.

[3] Erturk, A., Bilgen, O., Fotenille, M., and Inman, D.J., "Piezoelectric Energy Harvesting from Macro-Fiber Composites with an Application to Morphing Wing Aircrafts," Proceedings on the 19th International Conference of Adaptive Structures and Technologies, Monte Verita, Ascona, Switzerland, 6-9, Oct. 2008.

[4] De Marqui, Jr C., Vieria, W. G. R., Erturk, A., and Inman, D. J., "Modeling and Analysis of Piezoelectric Energy Harvesting from Aeroelastic Vibrations using the Doubletlattice method," ASME Journal of Vibration and Acoustics, Vol. 133, 2010, 011003.

[5] Tang, L., Paidoussis, M., and Jiang, J., "Cantilevered Flexible Plates in Axial Flow: Energy Transfer and the Concept of Flutter-mill," Journal of Sound and Vibration, Vol. 326, 2009, pp. 263-276.

[6] Pobering, S., Ebermeyer, S., and Schwesinger, N., "Generation of Electrical Energy Using Short Piezoelectric Cantilevers in Flowing Media, Proceedings of SPIE, Vol. 7288, 2009, 728807.

[7] Bryant, M. and Garcia, E., "Modeling and Testing of 
a Novel Aeroelastic Flutter Energy Harvester," Journal of Vibration and Acoustics, Vol. 133, 2011, 011010.

[8] Bryant M, Wolff, E., and Garcia, E., “Aeroelastic Flutter Energy Harvester Design: The Sensitivity of the Driving Instability to System Parameters," Vol. 20, 2011, 125017.

[9] Dunnmon, J. A., Stanton, S. C., Mann, B. P., and Dowell, E. H., "Power Extraction from Aeroelastic Limit Cycle Oscillations," Journal of Fluids and Structures, Vol. 27, 2011, 1182-1198.

[10] Sousa, V. C., de Melo Anicézio M, De Marqui, Jr C., and Erturk, A., "Enhanced Aeroelastic Energy Harvesting by Exploiting Combined Nonlinearities: Theory and Experiment," Smart Materials and Structures, Vol. 20, 2011, 094007.

[11] de Melo Anicézio, M., De Marqui, Jr C., Erturk, A., and Inman, D. J., "Linear and Nonlinear Aeroelastic Energy Harvesting using Electromagnetic Induction," Proceedings of the ASME 2011 Conference on Smart Materials, Adpative Structures and Intelligent Systems, SMASIS2011-5171, Sottsdale, Arizona, USA, 2011.

[12] Abdelkefi, A., and Hajj, M.R., "Performance enhancement of wing-based piezoaeroelastic energy harvesting through freeplay nonlinearity," Theoretical \& Applied Mechanics Letters, Vol. 3, 2013, 041001.

[13] Abdelkefi, A., and Nuhait, A.O., "Modeling and Performance analysis of cambered wing-based piezoaeroelastic energy harvesters," Smart Materials and Structures, Vol. 22, 2013, 095029 (14pp)., pp. 14.

[14] Bae, J.S., and Inman, D.J., "Aeroelastic Characteristics of Linear and Nonlinear Piezo-aeroelastic Energy Harvester," Journal of Intelligent Material System and Structures, Vol. 25, Vol. 4, 2014, (4), pp. 401-416.

[15] Bae, J. S., Inman, D. J., and Lee, I. "Effects of Structural Nonlinearity on Subsonic Aeroelastic Characteristics of Aircraft Wing with Control Surface," Journal of Fluids and Structures, Vol. 19, No. 6, 2004, pp. 747.763. 19(6): 747-763.

[16] Bae, J. S. and Lee, I. "Limit Cycle Oscillation of Missile Control Fin with Structural Non-linearity," Journal of Sound and Vibration, Vol. 269, 2004, pp. 669-687. 269: 669-687.

[17] Conner, M. D., Tang, D. M., Dowell, E. H., and Virgin, L. N., "Nonlinear behavior of a typical airfoil section with control surface freeplay: A numerical and experimental study," Journal of Fluid and Structures, Vol. 11, 1997, pp. 89109.

[18] Tang, L., Paidoussis, M., and Jiang, J., "Cantilevered Flexible Plates in Axial Flow: Energy Transfer and the Concept of Flutter-mill," Journal of Sound and Vibration, Vol. 326, 2009, pp. 326-276. 326: 263-276.

[19] Bae, J. S., Aeroelastic characteristics and flutter suppression considering structural nonlinearity, $\mathrm{Ph}$.D. Thesis, KAIST, Daejeon, Korea, 2002. .

[20] Kim, J.Y., Kim, K.S., and Lee, I., “Transonic Aeroelastic Analyses of Wings Considering Viscous and Thickness Effects," International Journal of Aeronautical and Space Science, Vol. 9. No. 2, 2008, pp. 34-40.

[21] Theodorsen, T. General Theory of Aerodynamic Instability and the Mechanism of Flutter, NACA Report 496, 1935. .

[22] Karpel, M., "Design for Active Flutter Suppression and Gust Alleviation using State-space Aeroelastic Modeling," Journal of Aircraft, Vol. 19, No. 3, 1982, pp. 221-227. 19 (3), 221-227.

\section{Appendix A}

The new variables $\left(T_{i}\right)$ in Eqs. (11)-(13) are presented as follows.

$$
\begin{aligned}
& T_{1}=-\frac{1}{3} \sqrt{1-c^{2}}\left(2+c^{2}\right)+c \cos ^{-1} c \\
& T_{3}=-\frac{1}{8}\left(1-c^{2}\right)\left(5 c^{2}+4\right)+\frac{1}{4}\left(7+2 c^{2}\right) c \sqrt{1-c^{2}} \cos ^{-1} c-\left(\frac{1}{8}+c^{2}\right)\left(\cos ^{-1} c\right)^{2} \\
& T_{4}=c \sqrt{1-c^{2}}-\cos ^{-1} c \\
& T_{5}=-\left(1-c^{2}\right)+2 c \sqrt{1-c^{2}} \cos ^{-1} c-\left(\cos ^{-1} c\right)^{2} \\
& T_{7}=\frac{1}{8} c \sqrt{1-c^{2}}\left(7+2 c^{2}\right)-\left(\frac{1}{8}+c^{2}\right) \cos ^{-1} c \\
& T_{8}=-\frac{1}{3}\left(1+c^{2}\right) \sqrt{1-c^{2}}+c \cos ^{-1} c \\
& T_{9}=\frac{1}{2}\left[\frac{1}{3}\left(\sqrt{1-c^{2}}\right)^{3}+a T_{4}\right] \\
& T_{10}=\sqrt{1-c^{2}}+\cos ^{-1} c \\
& T_{11}=\cos ^{-1} c(1-2 c)+\sqrt{1-c^{2}}(2-c) \\
& T_{12}=\sqrt{1-c^{2}}(2+c)-\cos ^{-1} c(2 c+1) \\
& T_{13}=\frac{1}{2}\left[-T_{7}-(c-a) T_{1}\right]
\end{aligned}
$$

\section{Appendix B}

The nondimensional lift and moments are

$$
\left\{\begin{array}{l}
-\bar{L} \\
\bar{M}_{\alpha} \\
\bar{M}_{\delta}
\end{array}\right\}=\left\{\begin{array}{l}
-\frac{b}{m U_{\infty}^{2}} L \\
\frac{b^{2}}{I_{\alpha} U_{\infty}^{2}} M_{\alpha} \\
\frac{b^{2}}{I_{\delta} U_{\infty}^{2}} M_{\delta}
\end{array}\right\}=\left[\begin{array}{lll}
Q_{\xi \xi} & Q_{\xi \alpha} & Q_{\xi \delta} \\
Q_{\alpha \xi} & Q_{\alpha \alpha} & Q_{\alpha \delta} \\
Q_{\delta \xi} & Q_{\delta \alpha} & Q_{\delta \delta}
\end{array}\right]\left\{\begin{array}{c}
|\xi| \\
|\alpha| \\
|\delta|
\end{array}\right\} e^{i \kappa \tau}
$$

where the components of the nondimensional AICs $[Q(k)]$ are 


$$
\begin{aligned}
Q_{\xi \xi} & =-\frac{1}{\mu}\left[-k^{2}+i k C(k)\right] \\
Q_{\xi \alpha} & =-\frac{1}{\mu}\left\{a k^{2}+2 C(k)+i k[1+(1-2 a) C(k)]\right\} \\
Q_{\xi \delta} & =-\frac{1}{\mu}\left\{\frac{T_{1}}{\pi} k^{2}+\frac{2}{\pi} T_{10} C(k)+i k\left[-\frac{T_{4}}{\pi}+\frac{T_{11}}{\pi} C(k)\right]\right\} \\
Q_{\alpha \xi} & =\frac{1}{\mu r_{\alpha}^{2}}\left[-a k^{2}+i 2 k\left(a+\frac{1}{2}\right) C(k)\right] \\
Q_{\alpha \alpha} & =\frac{1}{\mu r_{\alpha}^{2}}\left\{k^{2}\left(\frac{1}{8}+a^{2}\right)+2\left(a+\frac{1}{2}\right) C(k)+i k\left[\left(a-\frac{1}{2}\right)+2\left(\frac{1}{2}-a\right)\left(\frac{1}{2}+a\right) C(k)\right]\right\}
\end{aligned}
$$

$Q_{\alpha \delta}$$$
=\frac{1}{\mu r_{\alpha}^{2}}\left\{-\frac{1}{\pi}\left(T_{4}+T_{10}\right)-\frac{k^{2}}{\pi}\left[T_{7}+(c-a) T_{1}\right]+\frac{2}{\pi}\left(a+\frac{1}{2}\right) T_{10} C(k)\right.
$$$$
\left.-i \frac{k}{\pi}\left[T_{1}-T_{8}-(c-a) T_{4}-T_{11}\left(a+\frac{1}{2}\right) C(k)\right]\right\}
$$$$
Q_{\delta \xi}=\frac{1}{\pi \mu r_{\delta}^{2}}\left\{-k^{2} T_{1}-i k T_{12} C(k)\right\}
$$$$
Q_{\delta \alpha}=\frac{1}{\pi \mu r_{\delta}^{2}}\left\{2 k^{2} T_{13}-T_{12} C(k)-i k\left[-2 T_{9}-T_{1}+T_{4}\left(a-\frac{1}{2}\right)\right]-i k\left(\frac{1}{2}-a\right) T_{12} C(k)\right\}
$$$$
Q_{\delta \delta}=\frac{1}{\pi \mu r_{\delta}^{2}}\left\{-\frac{k^{2}}{\pi} T_{3}-\frac{1}{\pi}\left(T_{5}-T_{4} T_{10}\right)-\frac{1}{\pi} T_{10} T_{12} C(k)+i \frac{k}{2 \pi}\left[T_{4} T_{11}-T_{11} T_{12} C(k)\right]\right\}
$$ 